\title{
Chronic Plaque Psoriasis Exacerbated by Telmisartan Therapy: A Case Report
}

\author{
Amisha $^{1}$, Paras Malik ${ }^{* 1}$, Monika Pathania ${ }^{1}$, Vyas Kumar Rathaur ${ }^{2}$ \\ ${ }^{1}$ Department of Internal Medicine, All India Institute of Medical Sciences Rishikesh, 249201, India \\ ${ }^{2}$ Department of Paediatrics, Government Doon Medical College, Dehradun, India \\ Correspondence should be addressed to Paras Malik; parasmalikaiims@gmail.com
}

Received 28 September 2019;

Accepted 14 October 2019;

Published 02 November 2019

\begin{abstract}
Background: Psoriasis is an immune-mediated chronic inflammatory dermatosis with a complex interplay of environmental and genetic factors. Among the various documented triggers, drug-related psoriasis constitutes a distinct entity with exposure resulting in either induction or exacerbation of psoriasis. Here we describe the case of a 45-year-old female with telmisartan aggravated chronic plaque psoriasis previously well controlled on oral methotrexate.
\end{abstract}

Keywords: Angiotensin II receptor blockers, drug-induced, psoriasis, cutaneous drug reaction

\section{Introduction}

Psoriasis is a commonly encountered clinical entity with an estimated prevalence of $11 \%$ in adults and $1.3 \%$ in children ${ }^{[1]}$. This papulosquamous disease can be generalized or localized; having an acute, subacute, or chronic clinical course varying in severity over time. It has multifactorial etiologies with several implicated risk factors - pregnancy, alcohol, smoking, infections, emotional stress, sunlight, hypocalcemia, and drugs ${ }^{[2]}$.

Although several antihypertensives, antifungals, and corticosteroids are traditionally described associations for drugrelated psoriasis in the literature, Angiotensin II receptor blockers (ARBs) associated with psoriatic lesions are less common. Moreover, in daily clinical practice, the attention to drug-related psoriasis induction, exacerbations, or treatment resistance is limited. Increased clinical awareness regarding these associations will lead to early recognition and better treatment.

\section{Case Presentation}

A 45-year-old female, non-smoker, non-alcoholic, presented to the outpatient clinic for a regular follow-up visit for hypertension, hypothyroidism, and psoriasis. She was a known case of plaque psoriasis for the past eight years, which had been well-controlled with oral methotrexate. She was diagnosed with hypothyroidism one year back, for which she was on oral Levothyroxine $75 \mathrm{mcg}$ once a day. She had also been hypertensive for the past three years and was consuming oral amlodipine whose dosage had been increased from $2.5 \mathrm{mg}$ to $10 \mathrm{mg}$ a day over the last year. Persistently high blood pressure readings (>150/100 mm Hg) were recorded in the past two outpatient visits, despite complete adherence to medications and strict dietary and lifestyle changes. Hence, she was switched to oral telmisartan $40 \mathrm{mg}$ once daily. She was reviewed after one week and was noted to be normotensive with no active complaints.

A month after this change in regimen, the patient presented in the outpatient clinic with a flare of her psoriatic lesions, reporting new lesions on her body. There was no history of recent sore throat, runny nose, burning micturition, fever, cuts, stings, burns, vaccinations, recent stressors; no exposure to cold or dry weather, or excessive sunlight exposure.

On general examination, her vitals were stable. During skin examination, multiple, well-defined, discrete plaques were observed on bilateral arms, legs, thighs, forearms, back, and chest. These plaques had silvery scales, desquamation, surrounding induration with mild erythema. The skin adjacent to the lesions showed dryness, fissures and cracks [Figure 1]. 


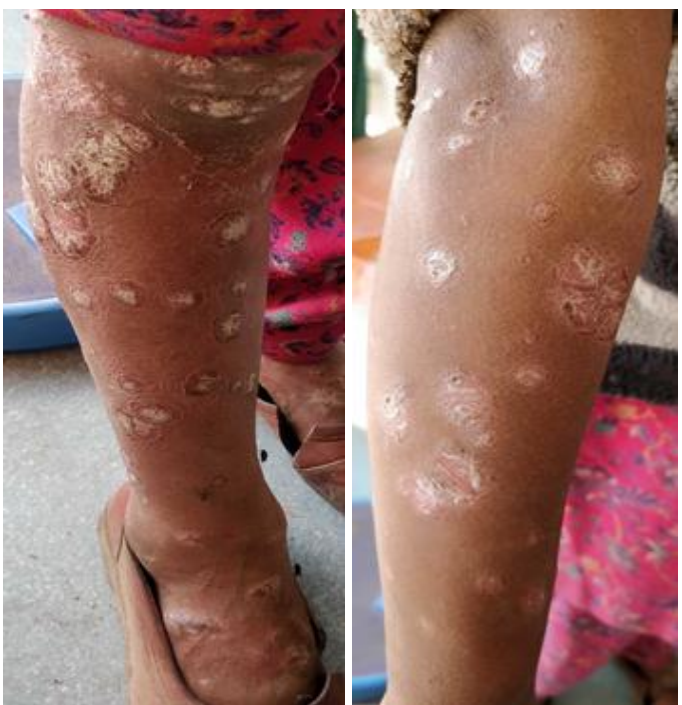

Figure 1: Psoriatic plaques with silvery scales with extensive leg \& elbow involvement.

The patient's urine pregnancy test was negative. Her random blood glucose, liver and renal function tests, complete blood count (CBC) with differential and serum electrolytes were found to be within the reference ranges. The Erythrocyte Sedimentation Rate (ESR) was 9 $\mathrm{mm} / 1 \mathrm{hr}$ (Normal: $0-15 \mathrm{~mm} / 1 \mathrm{hr}$ by Westergren method).

Based on the history, examination, and investigations, all the risk factors exacerbating psoriasis were ruled out. The patient was treated symptomatically, her Telmisartan was discontinued, and the patient was started on oral prazosin. Her skin lesions regressed gradually with complete resolution in a month. Hence, a diagnosis of Telmisartan-exacerbated plaque psoriasis was made based on the adverse effect profile of angiotensin-converting enzyme inhibitors (ACEIs) and angiotensin II receptor blockers (ARBs) described in the form of case reports in literature. Naranjo adverse drug reaction ${ }^{[3]}$ probability scale was the tool used to establish potential relation, and the relation calculated was probable for this case.

\section{Discussion}

Drug-related psoriasis has two major disease manifestations. Firstly, drugs can aggravate skin lesions in a person with preexisting disease (drug-aggravated psoriasis). Upon drug discontinuation, the lesions can either go in remission or persist. Secondly, drugs can initiate new lesions at an uninvolved site in a person with a history of psoriasis, or develop lesions in a person with no history of psoriasis (drug-induced psoriasis).

In clinical practice, medication-induced or exacerbated psoriasis is difficult to recognize. Firstly, there is a variable time gap between the initiation of drug administration and the onset of psoriasis, which is sometimes very long for certain drugs, making correlation difficult to establish. Secondly, there is little difference in the clinical and histological manifestations of drug-related and nondrug-related psoriasis. Thirdly, sometimes psoriatic flares persist even after the discontinuation of the offending drug, posing a clinical challenge. Fourthly, there is a lack of well-documented systematic analysis leading to lower strength of evidence. However, Naranjo adverse drug reaction ${ }^{[3]}$ probability scale can be used as a practical tool to establish potential relation.

Certain drugs have been strongly associated with psoriasis in various comprehensive reviews [Table 1]. Pustular psoriatic eruptions caused by angiotensin-converting enzyme (ACE) inhibitors like captopril ${ }^{[4]}$, enalapril ${ }^{[5]}$, and ramipril ${ }^{[6]}$ have been reported in the past. It was thought to be caused by the increased levels of bradykinin after inhibition of the angiotensin-converting enzyme by these drugs. In 2003 Kawamura et al. ${ }^{[7]}$ reported candesartan-induced pustular psoriasis. In 2011, Lamba et al. ${ }^{[8]}$ reported Losartan; and in 2015, Subramaniam Keerthi et al. ${ }^{[9]}$ reported Telmisartan as a cause of pustular psoriatic eruptions. ARBs are selective angiotensin receptor type 1 blockers which do not increase bradykinin levels challenging the above-proposed mechanism. The exact interplay of the kinin-kallikrein system and arachidonic pathway in psoriasis is yet to be established, but the above reports suggest that they do have a role to play.

The potential of Telmisartan to provoke psoriasis is important to clinically identify as there is increased utilization of this drug for control of hypertension and heart disease these days, owing to its cardio- and reno-protective effects ${ }^{[10]}$. Secondly, psoriatic patients are found to be at an increased risk of systemic comorbidities like hypertension and cardiovascular diseases ${ }^{[11]}$, and the likelihood of using ACE-Is in these patients is high.

Conflicts of Interest

This section is compulsory. A competing interest exists when professional judgment concerning the validity of research is influenced by a secondary interest, such as financial gain. We require that our authors reveal any possible conflict of interest in their submitted manuscripts. If there is no conflict of interest, authors should state that "The author(s) declare(s) that there is no conflict of interest regarding the publication of this paper."

\section{Funding Statement}

There was no funding acquired during any step of the manuscript preparation or publication process

\section{Acknowledgments}

None

\section{References}

[1] Michalck IM, Loring B, John SM. A systematic review of worldwide epidemiology of psoriasis. J Eur Dermatol Venereol. 2017;3: 2015-212.

[2] Griffiths CE, Barker JN. Psoriasis. In: Burns T, Breathnach S, Cox N, Griffiths C, editors. Rook's Textbook of Dermatology; 2012;44-54.

[3] Naranjo CA, Busto U, EM et al. A method for estimating the probability of adverse drug reactions. Clin Pharmacol Ther. 1981; 30: 239-245.

[4] Wolf R, Dorfman B, Krakowski A. Psoriasiform eruption induced by captopril and chlorthalidone. Cutis.1987; 40:162-4.

[5] Wolf R, Tamir A, Brenner S. Psoriasis related to angiotensin-converting enzyme inhibitors. Dermatologica.1990; 181:51-3.

[6] Thakor P, Padmanabhan M, Johnson A, Pararajasingam $\mathrm{T}$, Thakor S, Jorgensen W. Ramipril-induced generalized pustular psoriasis: case report and literature review. Am J Therapeutics.2010; 17:92-9.

[7] Kawamura A, Ochiai T. Candesartan cilexitil induced pustular psoriasis. Er J Dermatol.2003; 13: 406-7. 
[8] Lamba G, Palaniswamy C, Singh T, Shah D, Lal S, Vinnakota R et al. Psoriasis Induced by losartan therapy: A case report and review of literature. Am J Ther.2011; 18: 78-80.

[9] Subramaniam Keerthi, Murugauiyan Rangarai, Kaliaperumal Karthikeyan. Telmisartan aggravates pustular psoriasis. J pharmacother. 2015; 6: 107-109.
[10] Wienen W, Entzeroth M, van Meel JC, Stangier J, Busch $\mathrm{U}$, Ebner $\mathrm{T}$ et al. A review on telmisartan : A novel, long acting angiotensin II-receptor antagonist. Cardiovasc Drug Rev.2000; 18: 127-54.

[11] Catherine Ni, Melvin W Chiu. Psoriasis and comorbidities: links and risks. Clin Cosmet Investig Dermatol. 2014; 7: 119-132. 